\title{
Novel drugs in pediatric gliomas (Review)
}

\author{
DONGLI ZHANG, XIAOMING LIU, CONGHAI FAN and JIAO CHEN \\ Department of Neurology, Xuzhou Children's Hospital, Xuzhou, Jiangsu 221002, P.R. China
}

Received May 19, 2016; Accepted January 31, 2017

DOI: $10.3892 / \mathrm{ol} .2017 .5812$

\begin{abstract}
Astrocytomas (gliomas) are the most common primary brain tumors among adults and second most frequent neoplasm among children. New ideas and novel approaches are being explored world over with aim to devise better management strategeies for this deadly pathological state. We searched the electronic database PubMed for pre-clinical as well as clinical controlled trials reporting importance of various therapeutic drugs against gliomas. It was observed clearly that this approach of using therapeutic drugs is clearly evolving and has been observed to be promising future therapeutic avenue against gliomas. The searched literature on whole revealed that although gliomas are treated aggressively with surgery, chemotherapy and radiation, treatment resistance, drug toxicity and poor response rates among pediatric glioma patients, continue to drive the need to discover new and more effective chemotherapeutic agents. The present review is focused on the latest updates in therapeutic drugs against gliomas in pediatric patients. The important chemo-therapeutics discussed in this review included alkylating agents like temoxolomide, derivatives of platinum, nitrosoureas, topoisomerases, angiogenesis inhibitors and cytomegalovirus as therapeutic agents.
\end{abstract}

\section{Contents}

1. Introduction

2. Glioma therapy

3. Surgery

4. Radiotherapy

5. Chemotherapy

6. Alkylating agents

7. Derivatives of platinum

8. Nitrosoureas

9. Topoisomerase inhibitors

Correspondence to: Dr Conghai Fan, Department of Neurology, Xuzhou Children's Hospital, 18 Sudibei Road, Xuzhou, Jiangsu 221002, P.R. China

E-mail: fanconghai020@163.com

Key words: gliomas, brain, novel drugs
10. EGFR inhibitors

11. PDGFR- $\alpha$ inhibitors

12. Angiogenesis inhibitors

13. Cytomegalovirus as a therapeutic target in gliomas

14. Conclusion

\section{Introduction}

Pediatric gliomas as the name suggests, are childhood tumors of a glial origin with heterogeneous nature (1). Gliomas have discrete molecular individualities, varying clinical attributes and varying levels of malignancy. Their histological profile mirrors those of adult gliomas but the frequency of occurrence of distinct molecular signatures sets them apart from adult gliomas $(2,3)$. Such inherent differences in the biology of pediatric gliomas partly explain the reason for pediatric astrocytomas being unresponsive or having very low efficacies when administered with treatments tailored for adult gliomas.

\section{Glioma therapy}

The standard care of pediatric patients with gliomas involves the surgical resection of tumors coupled with adjuvant therapies such as radiation and chemotherapy, when relevant. Most often the choice of therapy is dictated by the patient's age, tumor location and tumor type. Patients with tumors having an indolent course such as optic pathway gliomas of Neurofibromatosis type 1 , may be restricted to observation only since approximately $50 \%$ of these tumors fail to progress further (4). However, other tumors require an aggressive treatment modality in order to improve patient survival.

\section{Surgery}

Surgery remains the principal treatment for well-circumscribed resectable pediatric high- and low-grade astrocytomas $(5,6)$. It is often recommended for tumors with distinct boundaries which are amenable to gross total or near total resection such as those that are peripherally located in the cerebellar and cerebral cortex (7). In general, surgical resection occastionally immensely decreases tumor volume, which is important to increase the efficacy of post-adjuvant treatments by radiation and/or chemotherapy on the residual unresected tumor. Surgical resection not only provides the tumorigenic tissues for histopathological diagnosis which is important for deciding follow-up therapies, but also decreases the size of the tumor 
mass, can prolong survival and sometimes can improve the overall quality of life for the patient (8).

With the exception of pilocytic astrocytomas, which have boundaries that are often well circumscribed, gliomas manifest predominantly a diffuse infiltrative behavior with no definitive boundaries. Therefore, complete resection is often not feasible even with the aid of sophisticated imaging and intraoperative techniques. Moreover, the surgical resection of brain tumors among children is often associated with the significant risk of morbidity and mortality. Examples of post-surgical complications include infections, bleeding, brain edema and stroke. Furthermore, neurological dysfunctions and even death can occur depending on which neighbouring functional neural networks at the resected tumor location are affected (9). Considering that some astrocytomas are located at eloquent regions of the brain where surgical resection may be more harmful than beneficial, and the fact that gross or even partial resection can never completely remove the tumor mass, other therapeutic options such as chemotherapy and radiotherapy must be utilized to improve the overall survival of the patient.

\section{Radiotherapy}

Radiotherapy is recommended as a first-line therapy for some unresectable pediatric gliomas such as diffuse intrinsic pontine gliomas (DIPG) or as a post-operative treatment for residual disease (10). Generally, the procedure involves the use of CT and MRI in order to define the tumor boundaries. This is then followed by the localized administration of 36-52 Gy photons (X-ray beams) in $1.8 \mathrm{~Gy}$ fractionations to the glioma mass of the pediatric patient. Over the years, radiotherapy has undergone dramatic improvements as a therapeutic option for patients with brain tumors. For instance, the introduction of 3D treatment planning protocols which allows for a $30-40 \%$ reduction in volume of normal brain parenchyma exposed to irradiation compared to the $2 \mathrm{D}$ planning approach (11), could theoretically reduce long-term morbidity and radiation associated sequelae. The use of rigid head immobilization and stereotactic irradiation approaches including stereotactic convergence therapy, fractionated stereotactic convergence therapy, fractionated conformal radiotherapy and intensity modulated radiotherapy can also permit the delivery of homogenously-focused irradiation fractionations to brain tumors of varying sizes, gradations and shapes (12). However, even though these advances have revolutionized radiotherapy, the physical property of X-rays including its high lateral side scatter and its toxic effect on normal tissues, which are proximal and distal to the targeted tumor mass, limits its use among pediatric patients who are younger than 3 years. Additionally, pediatric patients treated by radiotherapy are often at risk of developing secondary malignancies and often succumb to neurodevelopmental abnormalities including serious long-term neurocognitive and neuroendocrine sequelae.

\section{Chemotherapy}

Chemotherapy is a common and the most basic therapeutic module for any type of cancer and is often administered concomitantly with radiotherapy. It is usually recommended for children having disease progression after surgery and/ or radiotherapy, as well as for those who are too young and therefore have been deferred from radiotherapy because of its associated toxicities (13). However, it must be noted that, no current Food and Drug Administration (FDA)-approved chemotherapeutic agents are promisingly effective for pediatric astrocytomas; even when used in conjunction with other chemotherapeutic agents. Furthermore, dismal results still remain from current clinical trials; hence necessitating the identification and characterization of new chemotherapeutic agents for the treatment of pediatric gliomas. Below is a review of the efficacy and safety of the most common therapeutic agents used for the treatment of pediatric gliomas.

\section{Alkylating agents}

Akylating agents include analogues of nitrogen mustards, nitrosoureas, platinum derivatives, thiopeta, and so on (14). They generally function by chemically modifying DNA with an alkyl group or by inducing monoadducts, intrastrand and interstrand crosslinks, which attenuates DNA replication leading to cell cycle arrest and apoptosis (15). Summarized below, are the findings from clinical trials involving the most commonly used alkylating agents investigated to treat pediatric gliomas.

Temozolomide. The alkylating imidazotetrazine derivative, temozolomide, eventually became an FDA approved chemotherapeutic drug in 1999 for the experimental treatment of adult patients with refractory anaplastic astrocytomas (16). However, ongoing clinical trials, including the landmark phase III trial conducted by the European Organization for Research and Treatment of Cancer, unfortunately noted that the adjuvant post-operative use of temozolomide and radiotherapy only had a modest improvement in $<20 \%$ of the patients by increasing the median overall survival to 14.6 months for newly diagnosed glioblastoma patients, when compared to 12.1 months among similar patients treated by post-operative radiotherapy only $(17,18)$. Since 2005 , temozolomide has been the current recommended drug for the treatment of adult patients with glioblastoma multiforme (19). Temozolomide is administered orally, with a bioavailability of $96-100 \%$ and half-life of $1.8 \mathrm{~h}(20)$. At physiologic $\mathrm{pH}$, temozolomide is converted to a reactive intermediate known as 5-(3-methyl-1-triazeno)imidazole-4-carboxamide (MTIC) which subsequently methylates the $\mathrm{O} 6$ or N7-position of guanine as well as the N3-position of adenine. If left unrepaired by the cellular machinery, cells become susceptible to undergo apoptosis (21).

Clinical trials with temozolomide in pediatric glioma. Several clinical trials involving panels of pediatric glioma patients were conducted to assess the efficacy and toxicity of temozolomide used alone or concomitantly with other chemotherapeutic agents and radiotherapy (22). However, only few pediatric patients showed signs of complete/partial response or disease stabilization when treated with temozolomide. Furthermore, adverse side effects are noted with the use of temozolomide, including thrombocytopenia, nausea, emesis, fatigue, myelosuppression, sepsis, pneumonia and 
even death (23). Furthermore, a multicenter phase II clinical trial jointly conducted by the United Kingdom Children's Cancer Study Group and the French Society 20 for Pediatric Oncology showed only $12 \%$ of children with relapsed or progressive high-grade glioma, had a response to temozolomide administered daily at $200 \mathrm{mg} / \mathrm{m}^{2}$ for 5 consecutive days The response declines further to only $6 \%$, when the same treatment is administered to children with progressive diffuse intrinsic brainstem glioma (24). Due to the limited efficacy of using temozolomide alone for the treatment of pediatric gliomas, other clinical trials have examined the concomitant use of this drug with other therapeutic agents. For instance, a clinical trial involving 14 pediatric glioblastoma and anaplastic astrocytoma cases showed that the post-operative adjuvant use of temozolomide with lomustine resulted in only one complete response and one partial response (26).

The futile efficacy of temozolomide in treating pediatric gliomas may be attributed to a number of factors including an increase in the expression of the HOX genes, deficiency in the mismatch repair pathway, the absence of O6-methylguanine-DNA methyltransferase (MGMT) promoter methylation or even increased levels of MGMT protein expression in pediatric gliomas $(26,27)$. Several strategies are currently being investigated to sensitize pediatric gliomas to temozolomide; the most common being to therapeutically inactivate MGMT.

\section{Derivatives of platinum}

Carboplatin. The platinum derived compound, carboplatin is among the most commonly used chemotherapeutic agents for the treatment of pediatric gliomas (28). Recent results showed that, the 5-year overall survival rate for using carboplatin and vincristine was $48 \%$, which is significantly lower than $86 \%$ when pediatric low grade glioma patients were treated with thioguanine, procarbazine, lomustine and vincristine (29). Furthermore, children with DIPG and high grade gliomas did not show any significant improvements in overall survival when treated with carboplatin and etoposide or lobradimil. The use of other platinum-based drugs such as cisplatin in combination with etoposide (30) had initially suggested some encouraging results among pediatric glioma patients. Nonetheless, platinum-based drugs are also confounded with adverse side effects including nephrotoxicity with cisplatin (31) and hypersentivity with carboplatin (32), which limits their usefulness for treating pediatric patients with gliomas.

\section{Nitrosoureas}

Nitrosoureas function by establishing covalent interactions between DNA and proteins. This perturbs DNA repair, leading to cell cycle arrest and induction of apoptosis (33). Thus far, only carmustine (BCNU) and lomustine (CCNU), two nitrosoureas, have been clinically evaluated in pediatric glioma patients. For instance, a clinical trial conducted by the Children Cancer Group on 39 children diagnosed with malignant gliomas which examined the adjuvant use of eight drugs including BCNU, but no radiotherapy, noted a $24 \%$ response after two cycles of drugs with $51 \%$ of patients still being alive at 3 years (34). Recently, the French Society of Pediatric
Oncology noted a modest event-free 10-year survival rate of $13.3 \pm 9.4 \%$ in pediatric glioblastomas following the concomitant use of BCNU with cisplatin and VP16 (BCV) (33).

\section{Topoisomerase inhibitors}

Topoisomerases are required to establish and maintain the topology of DNA hence play pivotal roles during DNA replication, repair and transcription (36). Inhibition of topoisomerase in cancerous cells is therefore expected to affect the integrity of DNA, leading to the induction of cell death. Indeed, numerous inhibitors of topoisomerase I and II were developed (37), but only irinotecan, topotecan and etoposide were thus far examined in pediatric gliomas.

\section{EGFR inhibitors}

The epidermal growth factor receptor (EGFR) has materialized as an imperative target in the treatment of gliomas due to the fact that it is overexpressed in more than $40 \%$ of adult glioblastomas, and approximately $11 \%$ of pediatric glioblastomas (39). Recently, the Innovative Therapies for Children with Cancer European Consortium examined the efficacy and safety of the EGFR inhibitor, erlotinib, among children with recurrent or relapsed malignant gliomas, as well those with brainstem gliomas, using a dose of $125 \mathrm{mg} / \mathrm{m}^{2}$ per day adjuvantly with radiotherapy (40). However, this study had a disappointing outcome of progression free survival in only $28 \%$ of subjects (40). A clinical study by Yalon et al (41) also investigated the daily administration of the mTOR inhibitor, rapamycin. The study, however, failed to show any efficacy of these two drugs for the treatment of pediatric low grade gliomas in 17/19 patients (41).

\section{PDGFR- $\alpha$ inhibitors}

The platelet-derived growth factor receptor (PDGFR)- $\alpha$ is an amplified and overexpressed genes in pediatric high-grade glioma (42). However, its relevance as a therapeutic target in pediatric gliomas remains to be investigated.

\section{Angiogenesis inhibitors}

Angiogenesis is a process whereby new vascular networks sprout from existing blood vessels but can also occur following the process of intussusception (43), vascular mimicry (that is, the development of blood channel linings from de-differentiated tumor cells), or even through the recruitment of circulating endothelial cells. Recent studies have focus on bevacizumab (avastin), a monoclonal antibody against the VEGF protein, for the management of glioblastoma multiforme (44). Among pediatric gliomas, bevacizumab was investigated as a single agent which could clinically improve the neurological responses of children with diffuse intrinsic gliomas when administered alone (45).

\section{Cytomegalovirus as a therapeutic target in gliomas}

Initially referred to as infectious agents with no relevance to human tumors, viruses are now well recognized as critical 
mediators in the etiology and progression of human cancer (46). Consistent with the role of viruses in human tumors several studies have identified the human cytomegalovirus (HCMV) or its gene product in malignant gliomas $(47,48)$. Data supporting HCMV tumor promoting function is further reinforced by compelling in vivo evidence demonstrating reduced survival time in Mut3 glioma mouse models infected with mouse cytomegalovirus compared to mock infected or herpes simplex virus 1 infected control. Moreover, glioblastoma patients with low-grade CMV infection have a 20-month increase in median overall survival rate and $\sim 66.6$ months increased in a two-year survival rate compared to those with high-grade CMV infection (49).

Promising findings have also been obtained using immunotherapeutic approaches against CMV in glioblastoma (50). These studies have demonstrated that, vaccination of a glioblastoma patient with autologous tumor lysate-pulsed dendritic cells can result in substantial CMV-specific T-cell immune response (51). Similarly, adoptive transfer of cytomegalovirus-specific T-cells in combination with temozolomide has been shown to result in long-term disease-free survival in a patient with glioblastoma by Crough et al (50). These findings collectively suggest CMV is an important therapeutic target in glioblastoma. Thus, strategies with the aim of blocking CMV-mediated oncogenesis are potentially useful for the treatment of patients with CMV-positive malignant glioma.

\section{Conclusion}

Many therapeutic strategies are under investigation worldwide and most of them are being examined in different phases of clinical trials. However, application of these therapeutics as gold standard in pediatric gliomas remains to be implemented.

\section{References}

1. Wiese M, Schill F, Sturm D, Pfister S, Hulleman E, Johnsen SA and Kramm CM: No significant cytotoxic effect of the EZH2 inhibitor tazemetostat (EPZ-6438) on pediatric glioma cells with wildtype histone 3 or mutated histone 3.3. Klin Padiatr 228: 113-117, 2016.

2. Jones C, Perryman L and Hargrave D: Paediatric and adult malignant glioma: close relatives or distant cousins? Nat Rev Clin Oncol 9: 400-413, 2012.

3. Jones DT, Gronych J, Lichter P, Witt O and Pfister SM: MAPK pathway activation in pilocytic astrocytoma. Cell Mol Life Sci 69: 1799-1811, 2012.

4. Hargrave D: Paediatric high and low grade glioma: the impact of tumour biology on current and future therapy. Br J Neurosurg 23 351-363, 2009.

5. Khatua S, Moore KR, Vats TS and Kestle JR: Diffuse intrinsic pontine glioma-current status and future strategies. Childs Nerv Syst 27: 1391-1397, 2011

6. Pollack IF, Stewart CF, Kocak M, Poussaint TY, Broniscer A, Banerjee A, Douglas JG, Kun LE, Boyett JM and Geyer JR: A phase II study of gefitinib and irradiation in children with newly diagnosed brainstem gliomas: a report from the Pediatric Brain Tumor Consortium. Neuro Oncol 13: 290-297, 2011.

7. Mueller S and Chang S: Pediatric brain tumors: current treatment strategies and future therapeutic approaches. Neurotherapeutics 6: 570-586, 2009.

8. Hardesty DA and Sanai N: The value of glioma extent of resection in the modern neurosurgical era. Front Neurol 3: 140, 2012.

9. Moghrabi A, Friedman HS, Burger PC, Tien R and Oakes WJ: Carboplatin treatment of progressive optic pathway gliomas to delay radiotherapy. J Neurosurg 79: 223-227, 1993.

10. Urbański B: The future of radiation oncology: considerations of young medical doctor. Rep Pract Oncol Radiother 17: 288-293, 2012
11. Kortmann RD, Jeremic B and Bamberg M: Radiotherapy in the management of low-grade gliomas. In: Combined Modality Therapy for Central Nervous System Tumors. Petrowich Z, Brady LW, Apuzzo ML and Bamberg M (eds). Springer, Berlin, pp317-326, 2000.

12. Kortmann RD, Timmermann B, Taylor RE, Scarzello G, Plasswilm L, Paulsen F, Jeremic B, Gnekow AK, Dieckmann K, Kay S, et al: Current and future strategies in radiotherapy of childhood low-grade glioma of the brain. Part I: treatment modalities of radiation therapy. Strahlenther Onkol 179: 509-520, 2003.

13. Burzynski SR: Treatments for astrocytic tumors in children: current and emerging strategies. Paediatr Drugs 8: 167-178, 2006.

14. Preusser M, Haberler C and Hainfellner JA: Malignant glioma: neuropathology and neurobiology. Wien Med Wochenschr 156: 332-337, 2006.

15. Cruet-Hennequart S, Glynn MT, Murillo LS, Coyne S and Carty MP: Enhanced DNA-PK-mediated RPA2 hyperphosphorylation in DNA polymerase eta-deficient human cells treated with cisplatin and oxaliplatin. DNA Repair (Amst) 7: 582-596, 2008.

16. Mutter N and Stupp R: Temozolomide: a milestone in neuro-oncology and beyond? Expert Rev Anticancer Ther 6: 1187-1204, 2006.

17. Gállego Pérez-Larraya J, Ducray F, Chinot O, Catry-Thomas I, Taillandier L, Guillamo JS, Campello C, Monjour A, Cartalat-Carel S, Barrie M, et al: Temozolomide in elderly patients with newly diagnosed glioblastoma and poor performance status: An ANOCEF phase II trial. J Clin Oncol 29: 3050-3055, 2011.

18. Welzel G, Gehweiler J, Brehmer S, Appelt JU, von Deimling A, Seiz-Rosenhagen M, Schmiedek P, Wenz F and Giordano FA: Metronomic chemotherapy with daily low-dose temozolomide and celecoxib in elderly patients with newly diagnosed glioblastoma multiforme: A retrospective analysis. J Neurooncol 124: 265-273, 2015.

19. Cohen MH, Johnson JR and Pazdur R: Food and Drug Administration Drug approval summary: temozolomide plus radiation therapy for the treatment of newly diagnosed glioblastoma multiforme. Clin Cancer Res 11: 6767-6771, 2005.

20. Stupp R, Mason WP, van den Bent MJ, Weller M, Fisher B, Taphoorn MJ, Belanger K, Brandes AA, Marosi C, Bogdahn U, et al; European Organisation for Research and Treatment of Cancer Brain Tumor and Radiotherapy Groups; National Cancer Institute of Canada Clinical Trials Group: Radiotherapy plus concomitant and adjuvant temozolomide for glioblastoma. N Engl J Med 352: 987-996, 2005.

21. Hegi ME, Liu L, Herman JG, Stupp R, Wick W, Weller M, Mehta MP and Gilbert MR: Correlation of $\mathrm{O}^{6}$-methylguanine methyltransferase (MGMT) promoter methylation with clinical outcomes in glioblastoma and clinical strategies to modulate MGMT activity. J Clin Oncol 26: 4189-4199, 2008.

22. Jalali R, Raut N, Arora B, Gupta T, Dutta D, Munshi A, Sarin R and Kurkure P: Prospective evaluation of radiotherapy with concurrent and adjuvant temozolomide in children with newly diagnosed diffuse intrinsic pontine glioma. Int J Radiat Oncol Biol Phys 77: 113-118, 2010.

23. Kuo DJ, Weiner HL, Wisoff J, Miller DC, Knopp EA and Finlay JL: Temozolomide is active in childhood, progressive, unresectable, low-grade gliomas. J Pediatr Hematol Oncol 25: 372-378, 2003.

24. Lashford LS, Thiesse P, Jouvet A, Jaspan T, Couanet D, Griffiths PD, Doz F, Ironside J, Robson K, Hobson R, et al; United Kingdom Children's Cancer Study Group and French Society for Pediatric Oncology Intergroup Study: Temozolomide in malignant gliomas of childhood: a United Kingdom Children's Cancer Study Group and French Society for Pediatric Oncology Intergroup Study. J Clin Oncol 20: 4684-4691, 2002.

25. Jakacki RI, Yates A, Blaney SM, Zhou T, Timmerman R, Ingle AM, Flom L, Prados MD, Adamson PC and Pollack IF: A phase I trial of temozolomide and lomustine in newly diagnosed high-grade gliomas of childhood. Neuro Oncol 10: $569-576,2008$.

26. Gaspar N, Marshall L, Perryman L, Bax DA, Little SE, Viana-Pereira M, Sharp SY, Vassal G, Pearson AD, Reis RM, et al: MGMT-independent temozolomide resistance in pediatric glioblastoma cells associated with a PI3-kinase-mediated $\mathrm{HOX} /$ stem cell gene signature. Cancer Res 70: 9243-9252, 2010.

27. Fassan M, Tassone E, Onisto M, Perilongo G, D'Avella D and Gardiman MP: MGMT promoter methylation in pediatric high-grade gliomas. Childs Nerv Syst 27: 7-8, 2011. 
28. Perilongo G: Considerations on the role of chemotherapy and modern radiotherapy in the treatment of childhood low grade glioma. J Neurooncol 75: 301-307, 2005.

29. Ater JL, Zhou T, Holmes E, Mazewski CM, Booth TN, Freyer DR, Lazarus KH, Packer RJ, Prados M, Sposto R, et al: Randomized study of two chemotherapy regimens for treatment of low-grade glioma in young children: a report from the Children's Oncology Group. J Clin Oncol 30: 2641-2647, 2012.

30. Massimino M, Spreafico F, Cefalo G, Riccardi R, Tesoro-Tess JD, Gandola L, Riva D, Ruggiero A, Valentini L, Mazza E, et al: High response rate to cisplatin/etoposide regimen in childhood low-grade glioma. J Clin Oncol 20: 4209-4216, 2002.

31. Miller RP, Tadagavadi RK, Ramesh G and Reeves WB: Mechanisms of cisplatin nephrotoxicity. Toxins (Basel) 2: 2490-2518, 2010

32. Genc DB, Canpolat C and Berrak SG: Clinical features and management of carboplatin-related hypersensitivity reactions in pediatric low-grade glioma. Support Care Cancer 20 385-393, 2012.

33. Kann HE Jr, Kohn KW, Widerlite L and Gullion D: Effects of 1,3-bis(2-chloroethyl)-1-nitrosourea and related compounds on nuclear RNA metabolism. Cancer Res 34: 1982-1988, 1974.

34. Geyer JR, Finlay JL, Boyett JM, Wisoff J, Yates A, Mao L and Packer RJ: Survival of infants with malignant astrocytomas. A report from the Childrens Cancer Group. Cancer 75: 1045-1050, 1995.

35. Chastagner P, Kalifa C, Doz F, Bouffet E, Gentet JC, Ruchoux MM, Bracard S, Desandes E and Frappaz D; French Society of Pediatric Oncology (SFOP) Pilot Study: Outcome of children treated with preradiation chemotherapy for a high-grade glioma: results of a French Society of Pediatric Oncology (SFOP) Pilot Study. Pediatr Blood Cancer 49: 803-807, 2007.

36. Vos SM, Tretter EM, Schmidt BH and Berger JM: All tangled up: how cells direct, manage and exploit topoisomerase function. Nat Rev Mol Cell Biol 12: 827-841, 2011.

37. Salerno S, Da Settimo F, Taliani S, Simorini F, La Motta C, Fornaciari G and Marini AM: Recent advances in the development of dual topoisomerase I and II inhibitors as anticancer drugs. Curr Med Chem 17: 4270-4290, 2010.

38. Moukharskaya J and Verschraegen C: Topoisomerase 1 inhibitors and cancer therapy. Hematol Oncol Clin North Am 26: 507-525, 2012

39. Bax DA, Gaspar N, Little SE, Marshall L, Perryman L, Regairaz M, Viana-Pereira M, Vuononvirta R, Sharp SY, Reis-Filho JS, et al: EGFRvIII deletion mutations in pediatric high-grade glioma and response to targeted therapy in pediatric glioma cell lines. Clin Cancer Res 15: 5753-5761, 2009.
40. Geoerger B, Hargrave D, Thomas F, Ndiaye A, Frappaz D, Andreiuolo F, Varlet P, Aerts I, Riccardi R, Jaspan T, et al; ITCC (Innovative Therapies for Children with Cancer) European Consortium: Innovative therapies for children with cancer pediatric phase I study of erlotinib in brainstem glioma and relapsing/refractory brain tumors. Neuro Oncol 13: 109-118, 2011.

41. Yalon M, Rood B, MacDonald TJ, McCowage G, Kane R, Constantini S and Packer RJ: A feasibility and efficacy study of rapamycin and erlotinib for recurrent pediatric low-grade glioma (LGG). Pediatr Blood Cancer 60: 71-76, 2013.

42. Paugh BS, Broniscer A, Qu C, Miller CP, Zhang J, Tatevossian RG, Olson JM, Geyer JR, Chi SN, da Silva NS, et al: Genome-wide analyses identify recurrent amplifications of receptor tyrosine kinases and cell-cycle regulatory genes in diffuse intrinsic pontine glioma. J Clin Oncol 29: 3999-4006, 2011.

43. Hillen F and Griffioen AW: Tumour vascularization: sprouting angiogenesis and beyond. Cancer Metastasis Rev 26: 489-502, 2007.

44. Cohen MH, Shen YL, Keegan P and Pazdur R: FDA drug approval summary: bevacizumab (Avastin) as treatment of recurrent glioblastoma multiforme. Oncologist 14: 1131-1138, 2009.

45. Liu AK, Macy ME and Foreman NK: Bevacizumab as therapy for radiation necrosis in four children with pontine gliomas. Int $\mathrm{J}$ Radiat Oncol Biol Phys 75: 1148-1154, 2009.

46. Javier RT and Butel JS: The history of tumor virology. Cancer Res 68: 7693-7706, 2008.

47. Matlaf LA, Harkins LE, Bezrookove V, Cobbs CS and Soroceanu L: Cytomegalovirus pp71 protein is expressed in human glioblastoma and promotes pro-angiogenic signaling by activation of stem cell factor. PLoS One 8: e68176, 2013.

48. Penkert RR and Kalejta RF: Tale of a tegument transactivator: the past, present and future of human CMV pp71. Future Virol 7: 855-869, 2012.

49. Rahbar A, Stragliotto G, Orrego A, Peredo I, Taher C, Willems J and Söderberg-Naucler C: Low levels of human cytomegalovirus infection in glioblastoma multiforme associates with patient survival; - a case-control study. Herpesviridae 3: 3, 2012.

50. Crough T, Beagley L, Smith C, Jones L, Walker DG and Khanna R: Ex vivo functional analysis, expansion and adoptive transfer of cytomegalovirus-specific T-cells in patients with glioblastoma multiforme. Immunol Cell Biol 90: 872-880, 2012.

51. Prins RM, Cloughesy TF and Liau LM: Cytomegalovirus immunity after vaccination with autologous glioblastoma lysate. N Engl J Med 359: 539-541, 2008. 